\title{
Don't Get the Cart before the Horse: There Are No Shortcuts to Prescriptive Analytics
}

\author{
Jeremy Glassman \\ Arizona State University \\ Jeremy.Glassman@asu.edu
}

\author{
Benjamin Shao \\ Arizona State University \\ Benjamin.Shao@asu.edu
}

\author{
Robert St Louis \\ Arizona State University \\ St.Louis@asu.edu
}

\begin{abstract}
Davenport [5] argues that the most important component for putting big data into action within an organization is talent management, and this opinion is widely shared among academics. We interviewed the chief purchasing officers (CPOs) of 15 major corporations and found that they did not feel it was problematic to find the right people for data analytics teams, and did not feel it was difficult to get resources to support data analytics efforts. Instead, they were frustrated by data issues such as granularity, accuracy, and integration. They also were intimidated by what they perceived to be the requirements for prescriptive analytics, and generally had not progressed beyond descriptive analytics. This article summarizes the roadblocks that the CPOs encountered as they attempted to move from descriptive to predictive to prescriptive analytics, and presents a set of steps which must be followed if organizations are to move up the analytics hierarchy.
\end{abstract}

\section{Introduction}

Almost every article on business intelligence distinguishes among descriptive, predictive and prescriptive analytics [3] [4] [5] [9]. Those same articles generally present these as a hierarchy, with prescriptive at the top of the hierarchy. As a result, everyone seems to want to move to prescriptive analytics as soon as possible, even if they do not understand what prescriptive analytics is and can do. This means that they want self-service and actionable analytics, and they want to have these capabilities as soon as possible.

Unfortunately, it is not possible to jump directly to prescriptive analytics. Similar to Maslow's needs hierarchy, there is an analytics hierarchy, and one cannot move to a higher level in the hierarchy until the competencies in the level immediately below are at least somewhat satisfied. To see where organizations typically are positioned within the analytics hierarchy, and to see what is keeping them from moving up the hierarchy, we interviewed the chief procurement officers (CPOs) from 15 major companies in the United States and Europe. The research questions we are trying to answer are: 1) What roadblocks have companies encountered as they try to move from descriptive to predictive to prescriptive analytics? and 2) What practices have companies found to be the most effective for overcoming those roadblocks?

\section{Literature review}

In 1943 Maslow [8] published his famous motivational hierarchy of needs. The insight that Maslow offered was that some motivational needs take precedence over others, and that one cannot move to a higher level in the hierarchy unless the need immediately below that level is substantially satisfied. This insight prompted others to develop additional hierarchies, including an analytics hierarchy of needs for organizations, which is illustrated in Figure 1 from Shealy [9]. Similar to Maslow's model, each lower level is a necessary foundation for the level above it. If that foundation is weak or nonexistent, the organization will not be able to reap the benefits of the next level.

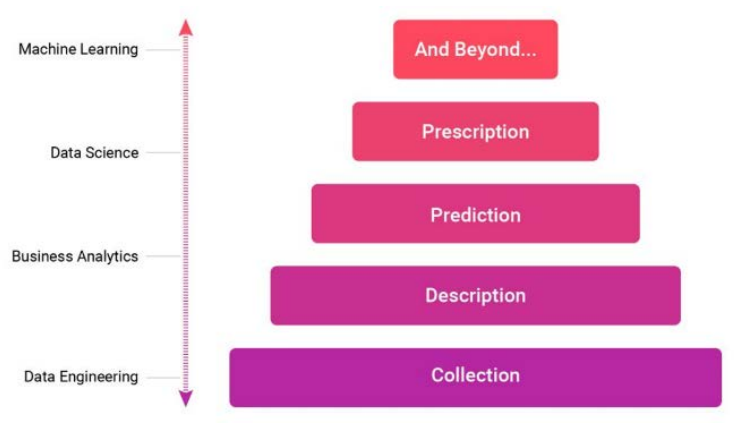

Figure 1. The analytics hierarchy of needs (source: Shealy [9])

Figure 1 shows that the foundation (level 1) of any data analytics program is effective data collection and management procedures. A company cannot analyze data that it does not have, and the quality of data analysis 
can be no greater than the quality of the data that has been collected. Robust procedures for data collection, cleaning, integration, governance, and retrieval must be in place before an organization can move to the next level of descriptive analytics. Our in-depth interviews with CPOs indicated that poor procedures for data collection, cleaning, and integration have resulted in about $80 \%$ of the data analytics effort being devoted to acquiring the needed data, and only $20 \%$ of the effort being devoted to analyzing the data itself. Moreover, poor data governance procedures have resulted in both the inability to integrate data and the storage of data at a granularity level unfit for the needed analyses.

After reliable data is collected and becomes available, it is possible to build dashboards and pivot tables. The dashboards enable managers to monitor key performance indicators in real time, and the pivot tables/data cubes enable managers to drill down on the data to better understand what drives performance. The ability to monitor performance broadly and quickly, and the ability to understand what drives performance, are essential for improving performance. However, in terms of the analytics hierarchy, this only reaches level two (descriptive analytics). Although most firms aspire to be at level four or five of the analytics hierarchy, our in-depth interviews with CPOs indicate very few firms have gotten beyond level two. Our interviews were designed to understand why so many organizations currently are stuck at level two.

The third level in the analytics hierarchy is referred to as predictive analytics. Predictive analytics uses models developed from past data to predict future actions, behaviors, or outcomes. For example, predicting buyer and supplier behavior is essential to predicting supply chain flows, and predicting supply chain flows can help to reduce both inventory and stockouts. Levels 1 and 2 of the analytics hierarchy provide the foundation for level three; since without data and an understanding of what drives performance, it is not possible to build a predictive model.

Overall, the success of predictive analytics projects relies on two components: 1) well-defined processes that standardize how data analysts should develop, test and deploy predictive models; and 2) a good set of predictive analytics techniques and tools that can be used to conduct the needed analyses. Ideally predictive analytics should be deployed as a virtuous cycle. That is, as soon as a new model is embedded in the business applications and processes to support decision-making and strategic planning, the analytics team should start the process again for a new analysis effort.

Level four in the analytics hierarchy is referred to as prescriptive analytics. "Prescriptive analyses are different from descriptive or predictive analyses in that they provide direct insight into the consequences of different actions by uncovering the key cause-and-effect relationships that impact the outcomes your organization cares about. ... Prescriptive analyses are about understanding what causes what, and why. While a predictive analysis aims to predict the value of an outcome of interest, a prescriptive analysis aims to understand the factors that determine that outcome, so that it can be influenced in the organization's favor" [3]. One of our CPOs put it very succinctly: "You gotta have predictive before you have prescriptive. We're working on predictive. That's the mode that we're in now. I want to get there so that five years from now we can get into the prescriptive analytics. And like I said earlier, knowing what will happen and knowing what to do are two different things."

Many practitioners as well as scholars seem unable to tell the difference between predictive and prescriptive analytics. Because similar models can be used for both predictive and prescriptive analytics, some have argued that it is a distinction without a difference [11].

We believe there is an important distinction between predictive and prescriptive analytics. Prescriptive analytics "attempts to quantify the effect of future decisions in order to advise on possible outcomes before those decisions are actually made. Prescriptive analytics not only predicts what will happen, but also tells why it will happen, and thereby provides recommendations regarding actions" [6]. A primary tool for prescriptive analytics, and one that is not used in predictive analytics, is experimentation. In this regard, Anderson and Simester [1] argue that "dissecting past data is a complicated task that few firms have the technical skills to master. Most companies will get more value from simple business experiments." In essence, they argue that the most effective form of prescriptive analytics is smart business experiments, and offer seven rules for conducting business experiments: 1) focus on individuals and think short term; 2) keep it simple; 3) start with a proof of concept; 4) slice the data; 5) try "out of the box" thinking; 6) measure everything that matters; and 7) look for natural experiments. These rules have strong implications for the types of data that should be collected, the types of persons that should be members of the analytics team, and the organizational culture that must be present.

An organization cannot move to prescriptive analytics until they have developed at least some competence in predictive analytics. The process of developing predictive models forces managers and analysts to consider relationships among factors. Moreover, until those relationships are known, it is not possible to design experiments to determine the effect of actions meant to manipulate those factors. One CPO told us: "We do not know why our predictive models are 
not working, and without that, we have no idea what actions to take."

Level five of the analytics hierarchy, aptly named "And Beyond," is a little more nebulous than the other levels. The theory is that through a combination of machine learning and embedded analytics, it is possible to continuously run experiments, and use the result of the experiments to adjust actions to improve operations. Theoretically, machine learning techniques can be applied to cleaned organizational data to run experiments and observe the impact on organizational performance of manipulating different inputs. However as Jeffrey Liker comments repeatedly in the Toyota Way [7], one cannot just automate a process. Management must first get the process under control. This sentiment also was echoed by several of the CPOs that we interviewed. Using big data and machine learning, it is possible to continuously run experiments and improve operations; but one can only do so after the processes are understood and under control.

Blum, Goldfarb, and Lederman [3] point out that closing the gap between the promise and reality of data analytics requires certain steps. These include: 1) focusing on the why and how of customer behavior, rather than the who, what, and which; 2) understanding the processes that generate the data; and 3) applying critical thinking to determine both what is valid evidence and what is relevant evidence. They argue that it is not possible to move from descriptive to predictive to prescriptive analytics without following these steps. Trist [10] and Baxter and Sommerville [2] further point out that that there is a limit to the productivity increase that can occur with a given technology in the absence of cultural change, and there also is a limit to the productivity increase that can occur with a given culture in the absence of technology change. To improve the performance of an organization, the 'social' and the 'technical' components must be brought together as interdependent parts of a socio-technical system, and simultaneously changed.

\section{CPO surveys}

As shown in the literature review, several very elegant strategies have been suggested for achieving data analytics maturity. But, as Winston Churchill pointed out: "However beautiful the strategy, you should occasionally look at the results." Toward that end, we conducted interviews with the CPOs of 15 major companies in the United States and Europe. Since complete anonymity was assured, we cannot provide the names of the companies. However, the 15 companies that we studied span 6 of the 20 major industry segments (30 percent), and 5,631,305 of the 16,049,223 (35 percent) of the business establishments covered by
NAICS. Thus they should provide a broad perspective on where organizations are with respect to data analytics maturity.

The goal of the interviews was to determine where organizations currently stand with respect to the data analytics hierarchy, what roadblocks organizations typically encountered as they try to move from descriptive to predictive to prescriptive analytics; and what practices companies have found to be the most effective for overcoming those roadblocks.

\subsection{Attained analytics levels}

Only one of the 15 firms that we interviewed was at level five of the hierarchy, and most had not progressed beyond level two. Table 1 shows the number of firms in our sample that were at each level, and the median annual sales for the firms at each level. Median sales are reported because each level had one firm that was an outlier on the high side for annual sales. Overall the distributions of sales for firms at level two and level three were quite similar, indicating that size did not help to explain the level of analytics maturity.

Table 1. Number of firms at each level of the hierarchy

\begin{tabular}{|l|c|c|}
\hline \multicolumn{1}{|c|}{ Level } & \# of Firms & $\begin{array}{c}\text { Median Sales (in } \\
\text { millions) }\end{array}$ \\
\hline And Beyond (L5) & 1 & $\$ 7,539$ \\
\hline Prescription (L4) & 0 & \\
\hline Prediction (L3) & 5 & $\$ 15,620$ \\
\hline Description (L2) & 9 & $\$ 12,497$ \\
\hline Collection (L1) & 0 & \\
\hline
\end{tabular}

We were surprised at how few of the organizations had progressed beyond level two. This might be due to the fact that we only studied the procurement function, and procurement could lag other functions such as category management or production. However, many of the CPOs stated that procurement did not lag the rest of the organization with respect to the use of analytics. Moreover, the problems that the CPOs experienced with respect to data granularity, accuracy, and governance likely extend to all functions within the organizations. Thus the roadblocks that CPOs experienced as they tried to move their function up the hierarchy, and the best practices that they used to overcome those roadblocks, should be relevant for all organizations.

\subsection{Typical analytics roadblocks}

This section describes the roadblocks to maturity that were identified by the CPOs we interviewed. To fully understand these roadblocks, it is necessary to 
understand the goals of CPOs. Thus we first identify the goals, and then describe the roadblocks. Each CPO had a slightly different way of expressing her/his goal. Due to space limitations, all of their comments cannot be reproduced here, but we do include four of the most relevant CPO comments in Table 2. We also show the analytics level of the CPO's firm (in parenthesis behind the comment). The analytics level of the firm is included to add context for the reader.

\section{Table 2. CPO comments on general goals}

So sourcing it is really just focused on two things: one is reducing input costs; and two is managing risk in a way that aligns with the business strategy. (L3)

Anybody in procurement who tells me competitiveness is not a top priority, l'd say you're dreaming. (L3)

Our primary focus is get the right product at the right time for our internal client. (L2)

Having a more coherent strategy around how does what we do help the business improve its performance. (L2)

As is evident from these comments, the overall goal of CPOs is to improve business performance by supporting the business strategy. To tie this more closely to analytics, we also asked how business analytics could help CPOs achieve their overall goals. Again each CPO had a slightly different way of expressing his/her hope and expectations for analytics teams. A few of their comments are reproduced in Table 3.

\section{Table 3. CPO expectations for data analytics}

Ultimately what l'd like to get to is to predict and anticipate somebody's purchase and actually present them - in more of an ordering system - that says, in the past when people like you bought this, this is the SLA that they agreed to. (L2)

Better drill down, faster turnaround, better visualization, improved capability to integrate a lot of data, all of those types of things. (L2)

We need to be warning our supply-chain colleagues about trends, risks, things that are potentially coming up. We should be able to tell you that this particular supplier may be defaulting. (L2)

Right now we're in a world of this is what we used to pay, this is a new price, this is what the savings is, and this is what the delta is between the old and the new. l'd really like to get to a point of this is what we're paying now, this is what it should cost, the opportunity to drive down the cost is in the transportation or in the fuel and labor, and to be able to hone in on that during negotiations. (L2)

Many of the CPOs' comments were industry specific, but they had some common threads. The dominant themes were for analytics to enable: 1) data integration, drill down, real time dashboards, and visualization; 2) determination of should-be pricing; 3) identification of opportunities to drive down the spend of major cost components such as transportation, fuel, and labor, and the ability to hone in on that during negotiations; 4) improved risk management; and 5) integration of internal information with external information to create more dynamic insights into the business. Unfortunately, most of the organizations that we interviewed have not progressed beyond data integration, drill down, real time dashboards, and visualization. The CPOs that we interviewed identified several roadblocks that are preventing organizations from moving up the analytics hierarchy.

One of the most frequently mentioned roadblocks to moving up the analytics hierarchy was an inappropriate level of granularity for the date that was captured and stored. A sample of the CPOs' comment on this issue are shown in Table 4.

\section{Table 4. CPO comments on data granularity}

When I did my competitive bid, what I found was we
had no coding on a purchase order to state whether
that PO was based on a competitive bid or not. (L2)
The agreement is typically a scanned PDF or maybe
an image file and you have trouble extracting out that
content into any analytics tool. (L2)
When it comes to terms and conditions, that is not
something that can be done using just data in the data
warehouses. Someone needs to manually look at
what has been agreed on and delivered. (L3)
We are working on access to logistics spend data.
But by the time we get it, it's so summarized that it's
almost not usable. (L2)

Because data governance is weak, and because CPOs have not been heavily involved with data governance, data frequently is not collected at the level of granularity that is needed for spend analysis and contract management. The only solution to this data granularity problem is a change in the process that is used for collecting and storing data.

A second roadblock that was identified by nearly every CPO was the difficulty of cleansing and integrating data. A sample of the CPOs' comments on this issue are presented in Table 5.

Table 5. CPO comments on data preparation

Takes them $80 \%$ of their time to get all the info together, and only $20 \%$ they ever spend on analyzing anything. (L2)

Many of these people know where to get stuff, but they have never been able to put it together before 
because it was such an arduous manual task to map all of that. And they don't have the time. (L2)

The gaps aren't in getting the data, the gaps are in the quality of data when we get it. The business process that creates the data in the first place is not there. It is not standardized. It is not consistent. (L3)

We don't capture everything we need. Some information gets lost between the various systems. Some data elements are not transferred out of the system or to the data warehouse. (L3)

These comments make it clear that many companies are struggling with data cleansing and integration. Most companies do not have standardized processes and standardized taxonomies for creating the data. As a result, they do not collect all of the needed data, and much of the data that they do collect is not accurate or consistent across systems.

A third and unexpected roadblock that we found was the reluctance of CPOs to even attempt to move to the top of the analytics hierarchy. Several CPOs simply were not comfortable with advanced analytics, and preferred not to operate in that space. Some comments that helped us see this are shown in Table 6.

\section{Table 6. CPO comments on advanced analytics}

Our proposal was: we're staying out of the advanced analytics space. (L2)

We want reports/dashboards and drill down for sales productivity, operations productivity, procurement productivity, HR issues, and legal issues. (L2)

The executive team, they want to go faster than technology or simple bandwidth will allow. So everybody talks these big fancy tools, but I would argue they are a little bit ahead of themselves. (L3)

We are probably getting closer to the predictive. We're using more pivot table based tools. I don't think we'll get into the latter, that's more what our center of excellence group would do. (L2)

Although only one CPO explicitly stated that he/she wanted to stay out of the analytics space, almost every CPO stated that the bulk of their effort was devoted to gathering data and descriptive analytics (levels one and two of the hierarchy), and only one CPO stated that his/her organization has progressed beyond predictive analytics (level 3 of the hierarchy shown in Figure 1). Our opinion is that when CPOs think about prescriptive analytics, they think in terms of complicated models rather than simple, easy to explain experiments. The one company that had reached level four and even level five of the hierarchy (prescriptive and beyond), was actively involved in running simple experiments and was using machine learning to facilitate this process. We discuss this further in the next section which focuses on how to remove the roadblocks.
Another roadblock that frequently was mentioned is the mindset of category managers. Several of the CPOs that we interviewed were very surprised at how difficult it is to get category managers to understand the value of data analytics. Some representative comments are produced in Table 7.

\section{Table 7. CPO comments on mindset issues}

That's been a struggle, getting people to understand the value of the data. I didn't anticipate that. (L2)

Try to find ways to communicate in their language, things that would make sense to them. Helping teams do trade-off analysis outside of sourcing has always been a little bit of a challenge. (L3)

I would say generally my biggest shock was the relative unsophistication of the buying teams in the space of analytics. (L3)

The hardest thing was getting the procurement team to recognize the benefits of the information. It allowed them to break down costs and have visibility. Getting them to buy into that was a huge challenge. (L5)

Closely related to the mindset of category managers is the overall culture of the organization. All of the CPOs agreed that culture is critical, and not having the support of top management is a very serious roadblock to getting the right culture. Some interesting comments in this regard are presented in Table 8.

\section{Table 8. CPO comments on culture}

The other one is the cultural shift, which means it is not just your leadership team supporting, but it is actually changing your metrics. How do you measure people? How do you drive the right behavior? Maybe instead of measuring a savings increase, you measure a through-put or cycle-time increase. (L3)

The problem is senior leadership and culture. Getting them to understand the additional benefit, getting them to understand that there is significant savings to be had. We just need to get past the "there's no time, there's no benefit" idea. (L3)

Before, the practitioner would think the focus of their job was to negotiate the best possible deal. It is, absolutely, but then how you negotiate it becomes important. Where do you capture your quotes? Where do you capture your RFP response? (L3)

The game changer for us is that now our top leaders understand the value that's there. And now instead of procurement trying to do small implementations on our own, now we've got this huge focus from our CEO on down that's actually investing money in making our landscape better. (L2)

Two general themes were prominent with respect to questions about whether the need to show the return on investment (ROI) is a roadblock for the use of analytics. 
The first is that demonstrating the ROI from investments in level two (descriptive) activities is easy to demonstrate, and tends to keep organizations from moving up to predictive and prescriptive activities when doing so is perceived as harder. The second is that when investment decisions are centralized, projects with relatively low ROIs never get funded, even if their potential return is substantial. Two very relevant comments about ROI are shown in Table 9.

\section{Table 9. CPO comments on required ROI}

We're driving a decent amount of ROI out of it, just being able to look across our regions, across vendors, do basic analytics. I am sure we will advance after we mine that lower hanging fruit. (L3)

In every company, getting incremental headcount is virtually impossible if you have no specific ROI that you can point to. In the centralized organization it's whoever's ROI is the highest are the people who get the resources. So if you have marginal ROls your project will never get addressed. (L3)

The most frequently mentioned tools were Tableau, HANA, SAP, Oracle, Tibco Spotfire, QED, Hadoop, Sales Force, Riva, QlikView, Hyperion, Watson, SAP Ariba, and Noetics. Some of these tools are used to put information into the data lake, some are used to get information out of the data lake, and some are used to create dashboards and visualizations. Almost all of the CPOs indicated the tools are not as user friendly as they would have liked to see. Some interesting comments about roadblocks associated with tools and vendors are presented in Table 10.

\section{Table 10. CPO comments on issues with tools}

But if you are a business person engaged in other activities, they become too complicated, and so there is a resistance and an unlikelihood of people using it. So ease of use, how intuitive is the interface is critical. (L3)

So your vendors, who are divorced from the end user experience, can never speak the language to explain what it is their tool can do. They always talk about it from a technology perspective, and that means nothing to a commodity manager sitting in Kuala Lumpur trying to make sure they're supporting manufacturing plants around Asia. (L3)

Almost all of the organizations had a hybrid structure for the analytics teams; with only one to three persons dedicated to analytics procurement, and much larger centralized analytics team. However the presence of those small dedicated procurement analytics teams was very important to the CPOs. Some comments that illustrate this point are shown in Table 11.

\section{Table 11. CPO comments on issues with analytics team structure}

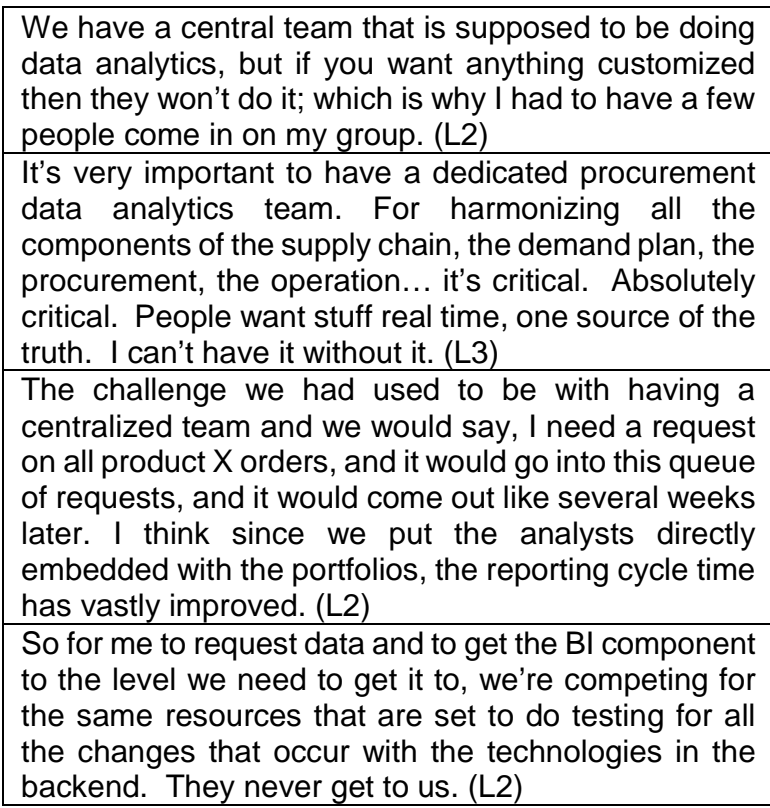

There also are a number of CPO comments about roadblocks that were very informative but did not fall into any of the major categories that we described above. These comments are presented in Table 12 .

\section{Table 12. Miscellaneous CPO comments on roadblocks}

\begin{tabular}{|c|}
\hline \\
\hline $\begin{array}{l}\text { We became the score keepers a little bit. And making } \\
\text { sure that we didn't become the scorekeeper was a } \\
\text { challenge. Making sure that we are looked at as a } \\
\text { team that you use to make your job better and easier } \\
\text { was something that was exciting. (L2) }\end{array}$ \\
\hline $\begin{array}{l}\text { Guys, you need to put some thought into a taxonomy } \\
\text { and indexing as these apps get created. We have to } \\
\text { figure out a way people can find an app that they're } \\
\text { after easily once there are } 5000 \text { apps out there. (L2) }\end{array}$ \\
\hline
\end{tabular}

Perhaps the most surprising of these miscellaneous comments was that none of the CPOs felt that it was challenging to find persons with the required skills. They also did not feel that it was challenging to get the budget required to hire those persons. This certainly is consistent with what we have observed when working with students on applied projects in our Master's program. There seem to be lots of projects where it is easy to demonstrate a high ROI for investments in analytics. 
It also is interesting to see that CPOs want to keep the focus of analytics on improving business operations, and not on preparing scorecards. This seems like a much more productive way to use analytics. Finally, it was informative to see CPOs recognize that a very large number of applications can be developed using analytics, and that those applications must be managed in order to reduce duplication and ensure reliability.

\subsection{Proactive Steps/ Best Practices}

One of the 15 firms that we studied was at level five of the analytic hierarchy and several others were at level three. This means it is possible to overcome the roadblocks identified in the prior subsection. The proactive steps that organizations took to overcome those roadblocks are described in this section.

The most commonly mentioned proactive step was the development of taxonomies for the data. Every CPO seemed to recognize that it is impossible to get clean data without taxonomies, and hence it is impossible to integrate data across platforms. Some comments of the CPOs on this topic are shown in Table 13.

\section{Table 13. CPO comments on taxonomies}

One of the best decisions we made was using the UN classifications for all our indirect spend. (L5)

We came up with a very practical and meaningful taxonomy of spend. (L2)

We wanted to compare apples to apples. We wanted to standardize in terms of currencies so we could see reporting by standard currency, and not budget currency. (L5)

So supply chain spearheaded a massive conference internally so that everybody codes material in the exact same way no matter where you are or what group you're in. (L2)

Although having a taxonomy is a necessary condition for success, it is far from being a sufficient condition. Unless a process is put in place to enforce use of the taxonomy, the data will not be collected or entered correctly. Some comments in this regard are presented in Table 14

\section{Table 14. CPO comments on the need for standard processes}

In order to standardize your data and get your data to a high level of quality and data integrity, standard processes common throughout all the businesses are critical. You can't standardize data when you have 25 different iterations of a process. (L2)

We have some pretty big investments going on right now to get our whole purchase to pay process onto one platform, which will then enable us to manage our data in a much better way. But with a company as big as we are, it's going to take us a couple years to get all that in place. (L2)

I tend to look at it more from a Toyota production system mentality, that you don't automate anything until you have debugged what your system is and you can do it effectively on a manual basis. (L3)

At this moment in time the roadmap is just to keep on piloting, and then once we are absolutely happy with everything then, we always industrialize the activity and in-house it to our shared service team. (L5)

Almost every CPO that we spoke with also stressed the importance of communicating clearly both within procurement and across the organization. This communication was essential for success at every level of the hierarchy. Some CPO comments in this regard are produced in Table 15.

\section{Table 15. CPO comments on communication}

Every month we meet and a different representative
does a presentation on how they use data in our
organization, where they pull the data from, and
where the pain points are with that data. (L2)
We pull together monthly analyst meetings so that
we're talking about and sharing best practices. (L2)
We do information sharing across the organization
and procurement. Enterprise-wide workshops on best
practices and tools and direction. (L3)
Our CIO and I, we talk every month. This was at my
request because we got so much stuff going on.
We're very collaborative with IT. IT is part of our cross
functional team. Not only from an IT perspective, but
a data security perspective. (L2)

CPOs also stressed the importance of choosing the right tools. Almost all of the CPOs commented that having the right tools is essential for both data quality and the widespread use of analytics. Some insightful comments are shown in Table 16.

\section{Table 16. CPO comments on tool selection}

We were very careful when we were choosing tools. With SpendHQ I didn't have to do hardly any manual intervention. The tool was pretty good out of the box. So when I got it, it was already probably $90 \%$ accurate in terms of getting the transaction in the right category that everybody agreed to. (L2)

So the level of the tools that might be utilized is one that's been a huge differentiator for us as users, for them to load data into pivot tools rather than on a spreadsheet, and allowing us to click on something and it dives into a group set, and be able to answer stuff at the point in time when we're looking rather 
than saying I wonder what happened here, and submit a report request. It's been fantastic. (L2)

What that has done is given us a way to display our metrics and our dashboards in a more user-friendly manner that people are now gravitating to because it's more user-friendly. (L2)

A valuable tool is machine learning - so the tool learns as you train it to recognize the transaction and where it goes. (L2)

The importance of interacting appropriately with the analytics team was another key proactive step. According to the CPOs that we interviewed, the best approach is not to ask analytics teams for data, but rather to ask them to help you solve a particular problem. Some insightful comments are shown in Table 17.

\section{Table 17. CPO comments on team interactions}

The other issue is not to tell them what we need them to pull, but what we're trying to solve. So having them become more of a team member and saying, how do we solve this, and using some of their creativity to come up with a solution. (L2)

If you haven't got a clear vision of where you're going, why you're going there, and what are the benefits you want to get out of it, I think you'll end up going down cul-de-sacs. (L5)

I always like to figure out - if we knew the answer to that, how would we drive value? (L5)

Integrating the business team into your business. Having them in your meeting so when you request data they know what you are requesting. (L2)

Another proactive step that was stressed by the CPOs that we interviewed was the importance of having one or two analytics persons dedicated to the procurement function. Some relevant CPO comments about this best practice are shown in Table 18.

\section{Table 18. CPO comments on need for dedicated procurement personnel}

We have a central team that is supposed to be doing data analytics, but if you want anything customized then they won't do it; which is why I had to have a few people come in on my group. (L2)

The challenge we had used to be with having a centralized team and we would say, I need a request on all product $X$ orders, and then it would go into this queue of requests, and it would come out like several weeks later. And I think since we put the analysts directly embedded with the portfolios, the reporting cycle time has vastly improved. (L2)

Although almost all of the CPOs that we interviewed felt it was possible, if not easy, to find people with the needed analytics skills, they also had strong opinions about what skills were needed. Some of their comments on the required skillset are shown in Table 19.

\section{Table 19. CPO comments on required skills}

I want some young, highly technical analysts who are well-versed in C++ or Python and understand the business because they came up through the ranks. (L3)

One of the things you need is somebody with the ability to have a foot in the business side but then, dangerously enough, a foot in the technical side. (L3) The person that can take a spreadsheet full of numbers and tell me these are the key ones, these are the important ones. (L2)

It's great that we have the basic analytical skills, that is helpful; but what's equally important, or even more important, is the business skills to say here's how we use this data to help us all make better decisions. (L2)

As we mentioned in the subsection on roadblocks, most of the CPOs were anxious about trying to move to levels four or five of the analytics hierarchy. Our explanation for this is that they equate levels four and five to the use of complex mathematical models, and they are not comfortable with those models. However, as Anderson and Simester [1] point out, the most effective form of prescriptive analytics is smart business experiments, not complicated analytics. Of the 15 organizations that we studied, only one had progressed to level five of the analytics hierarchy, and they did that by running simple, smart experiments. Some comments from the CPO of that company are shown in Table 20.

Table 20. CPO comments on experiments

One we're working on is we're looking at changing the process steps in the organization by doing experiments in different ways with 2 or 3 different suppliers in terms of reviewing contracts. (L5)

We have 2 experiments online at the moment to help us understand how we should react to the terms of a contract. Rather than everything having to go to a person, like it does today, we are using machine learning to help us deal with different scenarios. (L5)

Finally there were a number of comments about proactive steps that did not fit into a specific category, but nonetheless were very insightful. These miscellaneous comments are shown in Table 21.

\section{Table 21. Miscellaneous CPO comments on} Proactive Steps

Our typical way of thinking about prioritization, which is let's go find where we've got the biggest problem or 


\begin{tabular}{l} 
the biggest bang for the buck and let's attack that; I'm \\
not sure that theory necessarily works effectively in \\
this space. I look at where there are opportunities \\
where I can either break things down into bite-size \\
pieces that I can deal with. (L2) \\
\hline Having a finance person and having a supply chain \\
person on the analytics team, and watching us battle \\
out how we're going to pull data to present, has been \\
really, really helpful because we both have two \\
different perspectives. (L2) \\
\hline The more we use reports from the analytics teams, \\
the more pressure they have to make sure that data \\
is correct because we're using it to report to \\
leadership. (L2) \\
\hline When we engaged in some of these discussions with \\
suppliers, at first I assumed that there would be a lot \\
of resistance - wow, you're coming at me with all this \\
data. I think interestingly, one of the comebacks from \\
some of the suppliers has been "wow, can you share \\
that data with me?" Which is interesting. (L3)
\end{tabular}

These comments show the need to look at problems from new perspectives. As one CPO commented: “The type of mindset you need is to be open, recognize change, and see the effect of change is not doing the same thing as you did yesterday." This applies to how a company uses analytics, how it builds analytics teams, and how it interacts with partners.

\section{Summary and Conclusions}

From the interviews of CPOs, we learned that there are no shortcuts to prescriptive analytics. Instead, there is an ordered set of steps that must be taken by all organizations. If equipped with a clear roadmap and guidance, a company can maneuver through the hurdles and navigate to the destination. Challenges and opportunities abound along the way, and it requires the dedication and devotion of the leadership to reach the goal of having an effective data analytics team.

We also learned that it is important to distinguish real problems from imagined ones, so that executives know where to focus their attention and where they should allocate resources. For instance, while academics have stressed that the talent in data analytics appears to be in short supply, the CPOs that we interviewed indicated that getting such skills does not constitute a real problem. The same can be said about getting resources and support from the top executives, as now there is wide recognition of the importance and value of applying data analytics to business functions like procurement. On the other hand, some factors that appear ordinary and unexciting at first sight are likely to create real problems.

One such factor is data. The analytics team has to understand how they can locate, categorize and maintain data for sourcing and procurement. Because such data is dispersed and distributed across units and organizations, collecting it generally poses a challenge. By the same token, before the team can perform any analysis, data must be prepared, integrated and validated. Given the amount and variety of data to be processed, analytics teams find it challenging to integrate data into one central data lake. Many companies still rely on staff members to manually clean and integrate data. This approach is resource-intensive and does not scale. Automation and machine learning can increase the speed of data preparation, cleaning, integration and classification.

As shown in Table 1, most of the companies we studied currently are operating at the descriptive stage, but striving to advance to the predictive and prescriptive stages. It is important to understand that like humans following Maslow's motivational hierarchy of needs, a company must make sure that it has achieved maturity at the level of descriptive analytics before making investments to move up to predictive and prescriptive analytics.

To provide a roadmap for guiding the journey going forward, we summarize the steps into two specific paths that every company must simultaneously follow to assemble and organize a high-performance data analytics team. The first is a technical path, and the second is a cultural path. This is consistent with SocioTechnical Systems Theory, which argues that there is a limit to the productivity increase that can occur through technology use in the absence of cultural change, and there is a limit to the productivity increase that can occur through cultural change in the absence of technology change. The only way to remove these limits is to simultaneously change both culture and technology.

Figure 2 summarizes the technical steps that the CPOs identified as necessary conditions for progressing up the analytics hierarchy. Every CPO that we interviewed stressed that a data taxonomy must be developed, and a process must be instituted to ensure that the taxonomy is followed. They further stressed that automation and machine learning can be used to scale data collection and categorization. Once the data is in an enterprise data warehouse or data lake, it needs to be made accessible to analysts and managers. This will happen only if user friendly tools are available that allow data analytics teams to create dashboards, enable drill down, construct data visualizations, and provide self-service analytics capabilities to non-technical managers and support staff.

After the data are thoroughly understood, the organization can then move on to forecasting in order to better understand demand, risk, and opportunities in their business environment. However, as one CPO told us, "knowing what will happen and knowing what to do are two different things." In order to know what to do, 
organizations must run smart business experiments, and must use machine learning to embed the results of those experiment in their business processes.

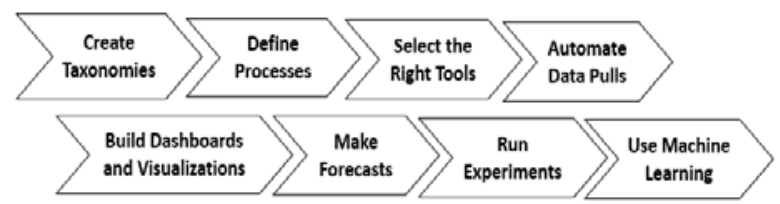

Figure 2. Technical path up the analytics hierarchy

Figure 3 summarizes the non-technical steps that the CPOs told us are necessary conditions for progressing up the analytics hierarchy. Every CPO that we interviewed stressed the importance of getting support from top management. One CPO summed it up very well when he said: "The game changer for us is that now our top leaders understand the value that's there." It is very difficult to move up the analytics hierarchy if top leaders are not convinced of the value of analytics. Every CPO also stressed the importance of close ties with the IT department. Personnel in the IT department best understand what data is available, what problems exist with the data, and how to extract the data. It is not useful to try and duplicate that expertise within the procurement or any other functional area of the organization.

Although getting top management on board and maintaining close ties with the IT department are important social steps, the most important "social" insight that we gained from the CPOs related to how managers should work with the analytics team. The comment that best summed this up for us was: managers should "not tell them what we need them to pull, but what we're trying to solve. So getting away from a tactical, descriptive definition, to having them become more of a team member and saying, how do we solve this, and using some of their creativity to come up with a solution." All of the CPOs agreed that just asking for data was a dead end street.

Finally, almost all of the CPOs mentioned that category managers struggle to understand how the information is going to help them build a better category strategy. For long term success, the CPOs stressed that it is crucial to convince category managers of the value of the data, and to publicize success that stemmed from the use of analytics throughout the organization.

$\left.\left.\left.\left.\left.\sum \begin{array}{c}\text { Get } \\ \text { Executive } \\ \text { Support }\end{array}\right\rangle \begin{array}{c}\text { Establish } \\ \text { Ties with } \\ \text { IT }\end{array}\right\rangle \begin{array}{c}\text { Define } \\ \text { the } \\ \text { Problem }\end{array}\right\rangle \begin{array}{c}\text { Work with } \\ \text { Category } \\ \text { Managers }\end{array}\right\rangle \begin{array}{c}\text { Spread } \\ \text { the Good } \\ \text { News }\end{array}\right\rangle$

Figure 3. Social path up the analytics hierarchy
The caveat is that there are no shortcuts on these paths, but the good news is that every company can proceed up the analytics hierarchy if they will just follow these steps. Each company has its own unique challenges, but following the best practices outlined by the CPOs that we interviewed will enable every company to leverage analytics to improve organizational performance.

\section{References}

[1] Anderson, E.T. and I. Simester, “A Step-by-Step Guide to Smart Business Experiments,” Harvard Business Review, March, 2011.

[2] Baxter, G. and I. Sommerville, "Socio-Technical Systems: From Design Methods to Systems Engineering,” Interacting with Computers, January, 2011

[3] Blum, B., A. Goldfarb, and M. Lederman, "The Path to Prescription: Closing the Gap Between the Promise and the Reality of Big Data," Rotman Management Magazine, Fall, 2015

[4] Davenport, Thomas H., “Analytics 3.0,” Harvard Business Review, December, 2013.

[5] Davenport, Thomas H., Big Data at Work: Dispelling the Myths, Uncovering the Opportunities, Harvard Business Review Press, 2014.

[6] Joshi, N., "Big Data Analytics: Descriptive vs. Predictive vs. Prescriptive,” Allerin https://www.allerin.com/blog/bigdata-analytics-descriptive-vs-predictive-vs-prescriptive, May 2, 2017.

[7] Liker, J. K., The Toyota Way: 14 Management Principles from the World's Greatest Manufacturer, McGraw-Hill, New York, 2004.

[8] Maslow, A. H., "A Theory of Human Motivation," Psychological Review, July, 1943.

[9] Shealy, D., “The Analytics Hierarchy of Needs,” DZone, https://dzone.com/articles/the-analytics-hierarchy-of-needs, March, 2016

[10] Trist, E., The Evolution of Socio-Technical Systems, Ontario Quality of Working Life Centre, Toronto, Canada, 1981

[11] Vorhies, B., "Prescriptive versus Predictive Analytics A Distinction without a Difference," Predictive Analytics Times,https://www.predictiveanalyticsworld.com/patimes/pr escriptive-versus-predictive-analytics-distinction-withoutdifference/4259/ November 14, 2014. 